\title{
Classification of acute pancreatitis-2012: revision of the Atlanta classification and definitions by international consensus
}

\author{
Peter A Banks, ${ }^{1}$ Thomas L Bollen, ${ }^{2}$ Christos Dervenis, ${ }^{3}$ Hein G Gooszen, ${ }^{4}$ \\ Colin D Johnson, ${ }^{5}$ Michael G Sarr, ${ }^{6}$ Gregory G Tsiotos, ${ }^{7}$ Santhi Swaroop Vege, \\ Acute Pancreatitis Classification Working Group
}

\begin{abstract}
- Additional data are published online only. To view these files please visit the journal online (http://dx.doi.org/10.1136/ gutjnl-2012-302779).

${ }^{1}$ Division of Gastroenterology, Hepatology, and Endoscopy, Harvard Medical School, Brigham and Women's Hospital, Boston,

Massachusetts, USA ${ }^{2}$ Department of Radiology, St Antonius Hospital, Nieuwegein, the Netherlands ${ }^{3}$ First Department of Surgery, Agia Olga Hospital, Athens, Greece

${ }^{4}$ Evidence-Based Surgery Research Unit, University of Nijmegen, Nijmegen, the Netherlands

${ }^{5}$ University Hospital

Southampton, Southampton, UK

${ }^{6}$ Department of Surgery, Mayo Clinic, Rochester, Minnesota, USA

${ }^{7}$ Division of Digestive Surgery, Metropolitan Hospital, Athens, Greece

${ }^{8}$ Department of Medicine, Division of Gastroenterology and Hepatology, Mayo Clinic Rochester, Minnesota, USA
\end{abstract}

\section{Correspondence to}

Michael G Sarr, Department of Surgery, Mayo Clinic (GU 10-01), 200 1st St SW, Rochester, MN 55905, USA; sarr.michael@mayo.edu

Revised 29 August 2012 Accepted 29 August 2012 Published Online First 25 October 2012

\section{GLinked}

- http://dx.doi.org/10.1136/ gutjnl-2012-303724 http://dx.doi.org/10.1136/ gutjnl-2012-303725 http://dx.doi.org/10.1136/ gutjnl-2012-304051

\section{ABSTRACT}

Background and objective The Atlanta classification of acute pancreatitis enabled standardised reporting of research and aided communication between clinicians. Deficiencies identified and improved understanding of the disease make a revision necessary.

Methods A web-based consultation was undertaken in 2007 to ensure wide participation of pancreatologists. After an initial meeting, the Working Group sent a draft document to 11 national and international pancreatic associations. This working draft was forwarded to all members. Revisions were made in response to comments, and the web-based consultation was repeated three times. The final consensus was reviewed, and only statements based on published evidence were retained.

Results The revised classification of acute pancreatitis identified two phases of the disease: early and late. Severity is classified as mild, moderate or severe. Mild acute pancreatitis, the most common form, has no organ failure, local or systemic complications and usually resolves in the first week. Moderately severe acute pancreatitis is defined by the presence of transient organ failure, local complications or exacerbation of co-morbid disease. Severe acute pancreatitis is defined by persistent organ failure, that is, organ failure $>48 \mathrm{~h}$ Local complications are peripancreatic fluid collections, pancreatic and peripancreatic necrosis (sterile or infected), pseudocyst and walled-off necrosis (sterile or infected). We present a standardised template for reporting CT images.

Conclusions This international, web-based consensus provides clear definitions to classify acute pancreatitis using easily identified clinical and radiologic criteria. The wide consultation among pancreatologists to reach this consensus should encourage widespread adoption.

\section{BACKGROUND}

The Atlanta Symposium in 1992 attempted to offer a global 'consensus' and a universally applicable classification system for acute pancreatitis. ${ }^{1}$ Although the Atlanta Classification has been useful, some of the definitions proved confusing. ${ }^{2}$ Better understanding of the pathophysiology of organ failure and necrotising pancreatitis and their outcomes, as well as improved diagnostic imaging, have made it necessary to revise the Atlanta Classification. This revision includes a clinical assessment of severity and

\section{Significance of this study}

What is already known on this subject?

- The original Atlanta Classification of acute pancreatitis of 1992 is outdated.

- Two types of acute pancreatitis have been described: acute oedematous pancreatitis and acute necrotising pancreatitis.

- The description of pancreatic and peripancreatic collections is confusing and not universal.

What are the new findings?

- This current global consensus classification of acute pancreatitis offers a comprehensive classification of acute pancreatitis, severity and peripancreatic collections.

- New information of aetiology, pathophysiology, severity and radiologic descriptions of pancreatic and peripancreatic collections are provided.

- This classification differentiates acute peripancreatic fluid, pancreatic pseudocyst, acute necrotic collections and walled-off necrosis.

How might it impact on clinical practice in the foreseeable future?

- This classification of acute pancreatitis will allow a consistent, worldwide classification.

- The description of pancreatic and peripancreatic collections on cross-sectional imaging will allow a consistent terminology across all studies.

- This classification of acute pancreatitis should avoid the confusion in terminology seen over the last 20 years.

provides more objective terms to describe the local complications of acute pancreatitis.

The goal of this report is to present the updated revision of the Atlanta Classification of acute pancreatitis in adults ( $>18$ years). This revision was designed to incorporate modern concepts of the disease, to address areas of confusion, to improve clinical assessment of severity, to enable standardised reporting of data, to assist the objective evaluation of new treatments, and to facilitate communication among treating physicians and between institutions. This consensus classification defines criteria for the diagnosis of acute pancreatitis, differentiates the two 
types of acute pancreatitis (interstitial oedematous pancreatitis and necrotising pancreatitis), classifies the severity of acute pancreatitis into three categories, and defines the morphology seen on imaging of pancreatic and peripancreatic collections that arise as complications of acute pancreatitis. This revision is not intended to be a management guideline.

\section{METHODS}

This classification was generated by an iterative, web-based consultation process led by a working group and incorporating responses from the members of 11 national and international pancreatic societies. All responses were reviewed by the working group, and the process was repeated by a web-based approach until the current fourth draft, which was then finalised for submission. A full description of the methods is shown in online supplementary appendix 1 . There are many substantial and important differences in the current document when compared to our preliminary working draft that appeared on the Pancreas Club website ${ }^{3}$ and which has been referred to by other authors. $^{4-8}$

\section{Revised definitions and classification of acute pancreatitis}

The following definitions and classifications are proposed for use in clinical and research communications.

\section{Definition of diagnosis of acute pancreatitis}

The diagnosis of acute pancreatitis requires two of the following three features: (1) abdominal pain consistent with acute pancreatitis (acute onset of a persistent, severe, epigastric pain often radiating to the back); (2) serum lipase activity (or amylase activity) at least three times greater than the upper limit of normal; and (3) characteristic findings of acute pancreatitis on contrast-enhanced computed tomography (CECT) and less commonly magnetic resonance imaging (MRI) or transabdominal ultrasonography. ${ }^{9-13}$

If abdominal pain suggests strongly that acute pancreatitis is present, but the serum amylase and/or lipase activity is less than three times the upper limit of normal, as may be the case with delayed presentation, imaging will be required to confirm the diagnosis. ${ }^{13} 14$ If the diagnosis of acute pancreatitis is established by abdominal pain and by increases in the serum pancreatic enzyme activities, a CECT is not usually required for diagnosis in the emergency room or on admission to the hospital.

\section{Definition of onset of acute pancreatitis}

The onset of acute pancreatitis is defined as the time of onset of abdominal pain (not the time of admission to the hospital). The time interval between onset of abdominal pain and first admission to the hospital should be noted. When patients with severe disease are transferred to a tertiary hospital, the intervals between onset of symptoms, first admission and transfer should be noted. Data recorded from a tertiary care hospital should be stratified to allow separate consideration of the outcomes of patients who were admitted directly and those admitted by transfer from another hospital (see online supplementary appendix 2 for suggested recording of data).

\section{Definition of types of acute pancreatitis}

Acute pancreatitis can be subdivided into two types: interstitial oedematous pancreatitis and necrotising pancreatitis.

\section{Interstitial oedematous pancreatitis}

The majority of patients with acute pancreatitis have diffuse (or occasionally localised) enlargement of the pancreas due to inflammatory oedema. On CECT, the pancreatic parenchyma shows relatively homogeneous enhancement, and the peripancreatic fat usually shows some inflammatory changes of haziness or mild stranding. There may also be some peripancreatic fluid (see below, Definition of pancreatic and peripancreatic collections) (figures 1 and 2). The clinical symptoms of interstitial oedematous pancreatitis usually resolve within the first week. ${ }^{15}$

\section{Necrotising pancreatitis}

About $5-10 \%$ of patients develop necrosis of the pancreatic parenchyma, the peripancreatic tissue or both (see below, Definition of pancreatic and peripancreatic collections) (figures 3, 4, 5). Necrotising pancreatitis most commonly manifests as necrosis involving both the pancreas and peripancreatic tissues and less commonly as necrosis of only the peripancreatic tissue, and rarely of the pancreatic parenchyma alone.

The impairment of pancreatic perfusion and signs of peripancreatic necrosis evolve over several days, ${ }^{16-19}$ which explains why an early CECT may underestimate the eventual extent of pancreatic and peripancreatic necrosis. In the first few days of the illness, the pattern of perfusion of the pancreatic parenchyma as seen on CECT may be patchy, with variable attenuation before the area of impaired enhancement becomes more demarcated and/or confluent. After the first week of the disease, a non-enhancing area of pancreatic parenchyma should be considered to be pancreatic parenchymal necrosis.

In peripancreatic necrosis, the pancreas enhances normally on CECT as it does with interstitial oedematous pancreatitis, but the peripancreatic tissues develop necrosis. Patients with peripancreatic necrosis alone have increased morbidity and intervention rates compared to patients with interstitial oedematous pancreatitis. 151720

The natural history of pancreatic and peripancreatic necrosis is variable, because it may remain solid or liquefy, remain sterile or become infected, persist, or disappear over time.

\section{Infected pancreatic necrosis}

Pancreatic and peripancreatic necrosis can remain sterile or become infected; most of the evidence suggests no absolute correlation between the extent of necrosis and the risk of infection and duration of symptoms. ${ }^{21-24}$ Infected necrosis is rare during the first week. ${ }^{21} 25$

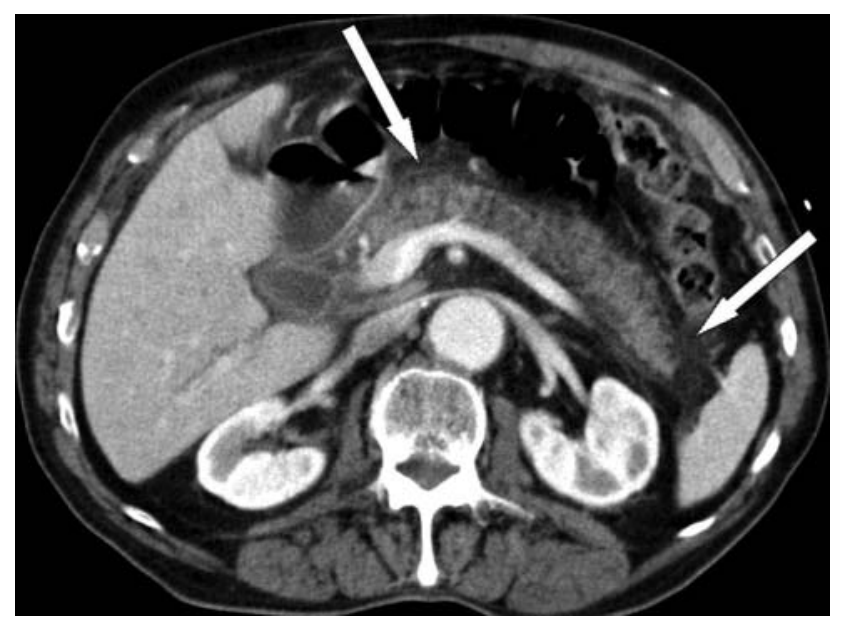

Figure 1 A 63-year-old man with acute interstitial oedematous pancreatitis. There is peripancreatic fat stranding (arrows) without an acute peripancreatic fluid collection; the pancreas enhances completely but has a heterogeneous appearance due to oedema. 

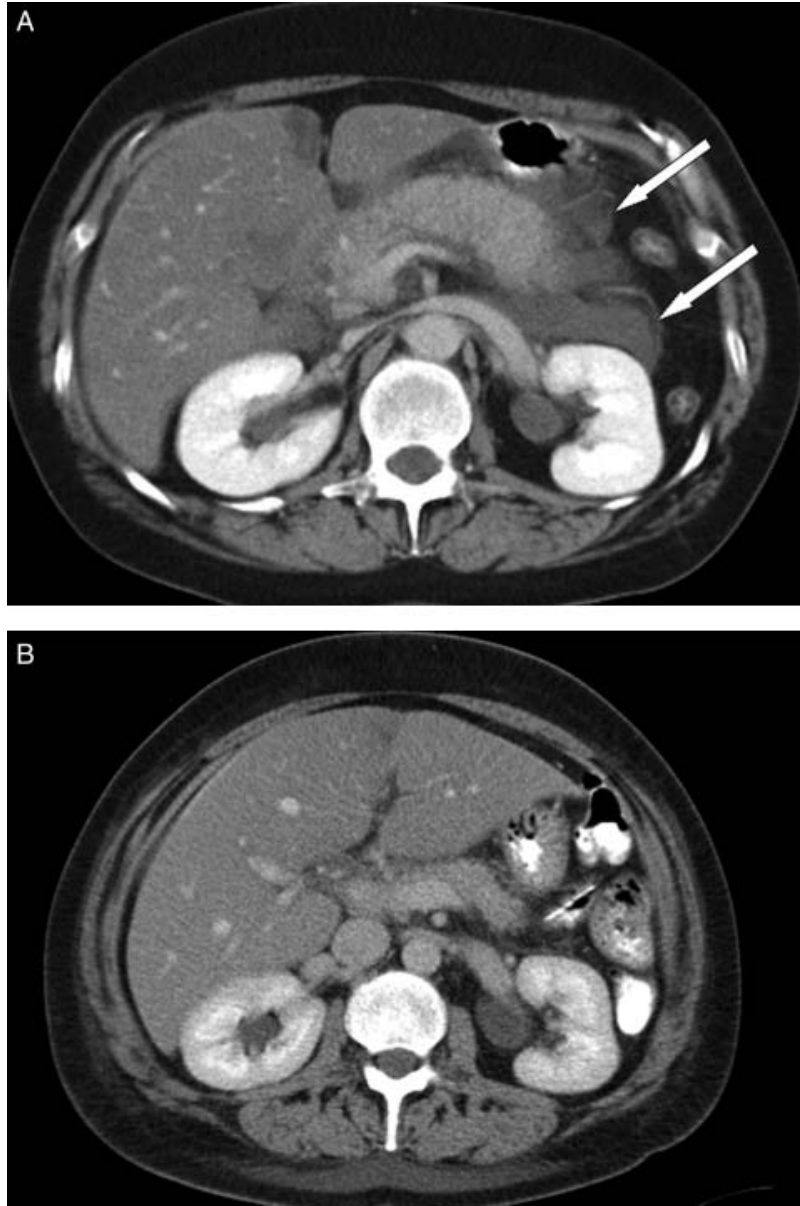

Figure 2 (A) A 38-year-old woman with acute interstitial oedematous pancreatitis and acute peripancreatic fluid collection (APFC) in the left anterior pararenal space (white arrows showing the borders of the APFC). The pancreas enhances completely, is thickened, and has a heterogeneous appearance due to oedema. APFC has fluid density without an encapsulating wall. (B) A few weeks later, a follow up CT shows complete resolution of the APFC with minimal residual peripancreatic fat stranding.

The diagnosis of infected pancreatic necrosis is important because of the need for antibiotic treatment and likely active intervention. $^{22}$ The presence of infection can be presumed when there is extraluminal gas in the pancreatic and/or peripancreatic tissues on CECT (figure 6) or when percutaneous, image-guided, fine-needle aspiration (FNA) is positive for bacteria and/or fungi on Gram stain and culture. ${ }^{26}$ There may be a varying amount of suppuration (pus) associated with the infected pancreatic necrosis, and this suppuration tends to increase with time with liquefaction. The original Atlanta Classification proposed the term 'pancreatic abscess' to define a 'localised collection of purulent material without significant necrotic material.'. This finding is extremely uncommon, and because the term is confusing and has not been adopted widely, ${ }^{27}$ the term 'pancreatic abscess' is not used in the current classification.

The development of secondary infection in pancreatic necrosis is associated with increased morbidity and mortality. ${ }^{28}$

\section{Complications of acute pancreatitis}

Definition of organ failure

Three organ systems should be assessed to define organ failure: respiratory, cardiovascular and renal. Organ failure is defined as
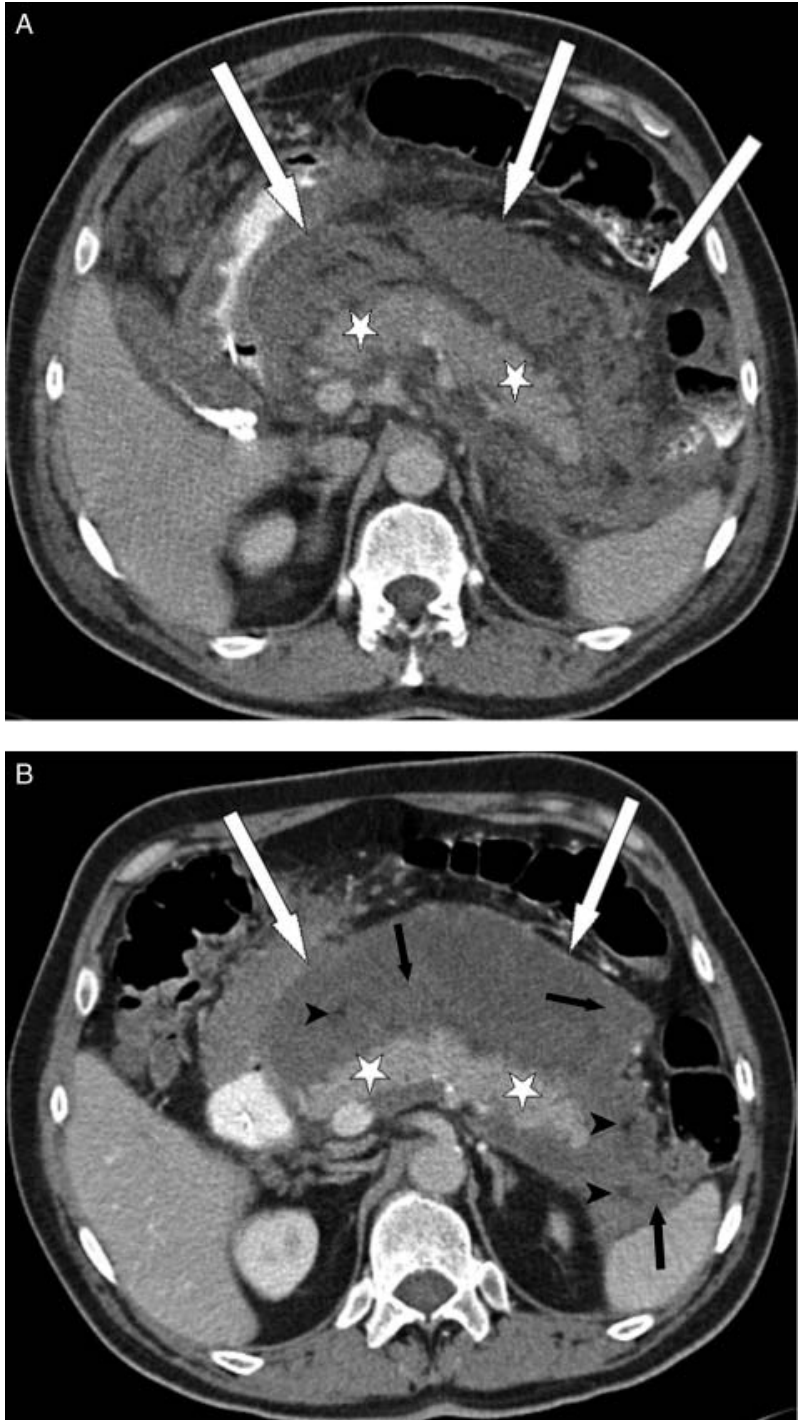

Figure 3 (A) Acute necrotic collections (ANC) in a 44-year-old man with acute necrotising pancreatitis involving only the peripancreatic tissues. Note enhancement of the entire pancreatic parenchyma (white stars) and the heterogeneous, non-liquid peripancreatic components in the retroperitoneum (white arrows pointing at the borders of the ANC). (B) The ANC in the same patient as (A) but imaged a few weeks later demonstrate a heterogeneous collection with areas of fat (black arrowheads) surrounded by fluid density, and areas which have a slightly greater attenuation (black arrows) than seen in collections without necrosis such as shown in figure 7 . This finding is typical for peripancreatic necrosis. White arrows denote border of ANC; white stars denote enhancement of pancreatic parenchyma. The ANC are not yet fully encapsulated.

a score of 2 or more for one of these three organ systems using the modified Marshall scoring system ${ }^{29}$ (table 1). The modified Marshall scoring system has the merit of simplicity, universal applicability across international centres, and the ability to stratify disease severity easily and objectively. ${ }^{10}$ The modified Marshall scoring system is preferred to the SOFA scoring system, ${ }^{30}$ which is for patients managed in a critical care unit and which takes into account the use of inotropic and respiratory support. Both scoring methods have the advantage of being able to be used on presentation and repeated daily. ${ }^{30} 31$ They also allow stratification of the severity of organ failure, although that is not part of the current classification. 

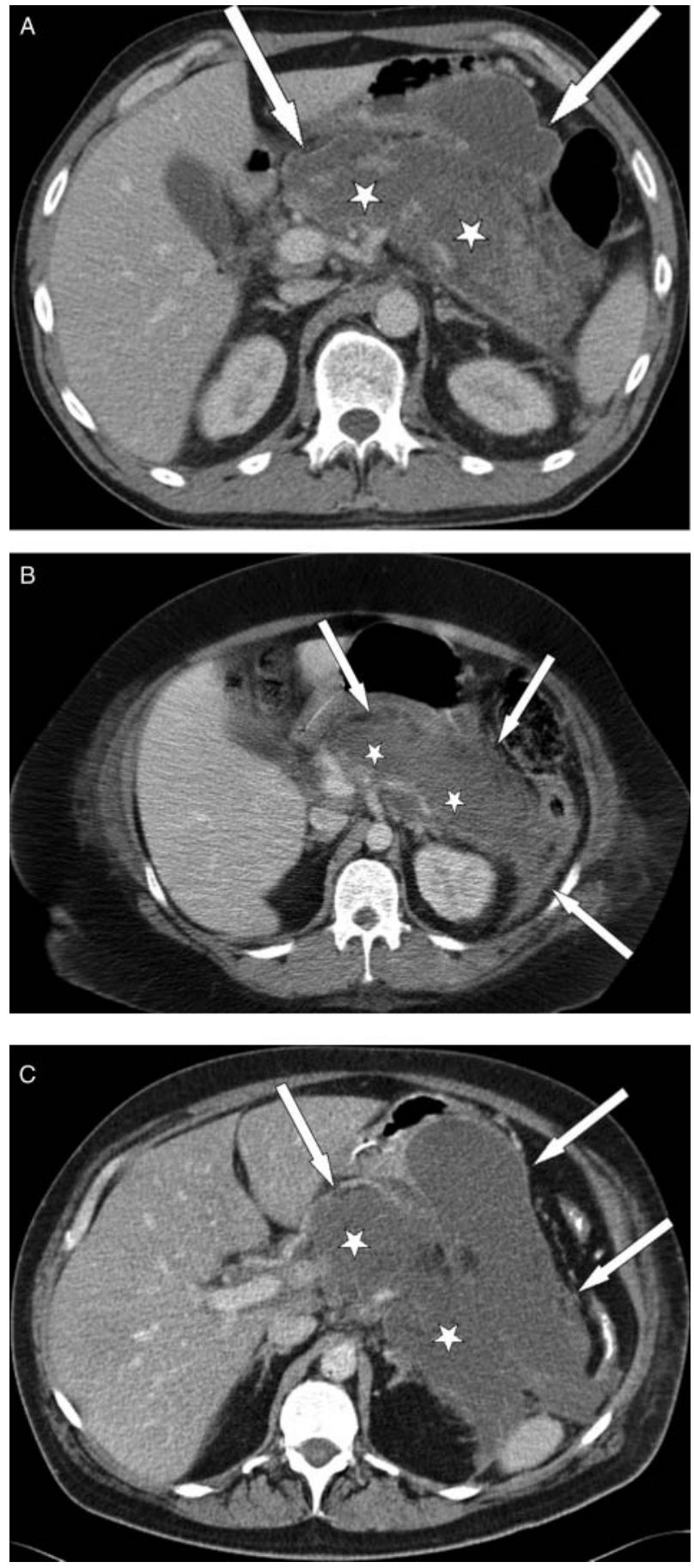

Figure 4 Three different patients $(A, B, C)$ with acute necrotising pancreatitis and acute necrotic collections (ANC) involving the pancreatic parenchyma and the peripancreatic tissues. In all three patients, there is extensive parenchymal necrosis (white stars) of the body and tail of the pancreas. Heterogeneous collections are seen in the pancreatic and peripancreatic tissues (white arrows pointing at the borders of the ANC) of the left anterior pararenal space $(A, B, C)$ and in the lesser sac $(A, C)$. These latter collections represent peripancreatic necrosis.

Definition of local complications

The original Atlanta Classification ${ }^{1}$ distinguished between uncomplicated interstitial pancreatitis and acute pancreatitis associated with 'local complications'. This distinction (local complications being absent or present) is useful. The natural

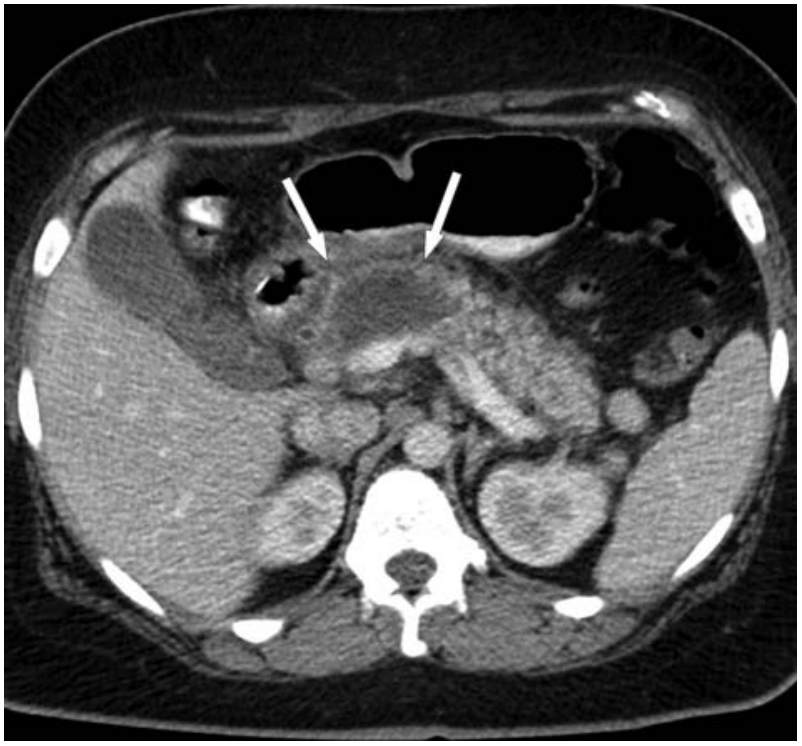

Figure 5 Acute necrotic collection (ANC) in a 47-year-old woman with acute necrotising pancreatitis involving the pancreatic parenchyma alone. Thin white arrows denote a newly developed, slightly heterogeneous collection in the region of the neck and body of the pancreas, without extension in the peripancreatic tissues.

history and clinical consequences of different local complications are now better understood and described. Local complications are acute peripancreatic fluid collection, pancreatic pseudocyst, acute necrotic collection and walled-off necrosis. The morphologic features of these local complications are described in detail later in this document (see below, Definition of pancreatic and peripancreatic collections). Other local complications of acute pancreatitis include gastric outlet dysfunction, splenic and portal vein thrombosis, and colonic necrosis.

Local complications should be suspected when there is persistence or recurrence of abdominal pain, secondary increases in serum pancreatic enzyme activity, increasing organ dysfunction, and/or the development of clinical signs of sepsis, such as fever and leucocytosis. These developments usually prompt imaging to detect local complications. The morphologic features of acute pancreatitis are well delineated by high resolution, multi-detector CECT and form the basis of the new, more objective definitions for the local complications of acute pancreatitis (box 1).

Pancreatic and peripancreatic collections should be described on the basis of location (pancreatic, peripancreatic, other), the nature of the content (liquid, solid, gas), and the thickness of any wall (thin, thick). The pattern and extent of impaired pancreatic parenchymal perfusion, if present, should also be described. ${ }^{27}$ The morphologic description of local complications is necessary for accurate diagnosis. Local complications alone, however, do not define the severity of acute pancreatitis (see below, Definition of severity of acute pancreatitis). ${ }^{32} 33$

\section{Definition of systemic complications}

Exacerbation of pre-existing co-morbidity, such as coronary artery disease or chronic lung disease, precipitated by the acute pancreatitis is defined as a systemic complication. In this document, we distinguish between persistent organ failure (the defining feature of severe acute pancreatitis) and other systemic complications, which are an exacerbation of pre-existing co-morbid disease. 


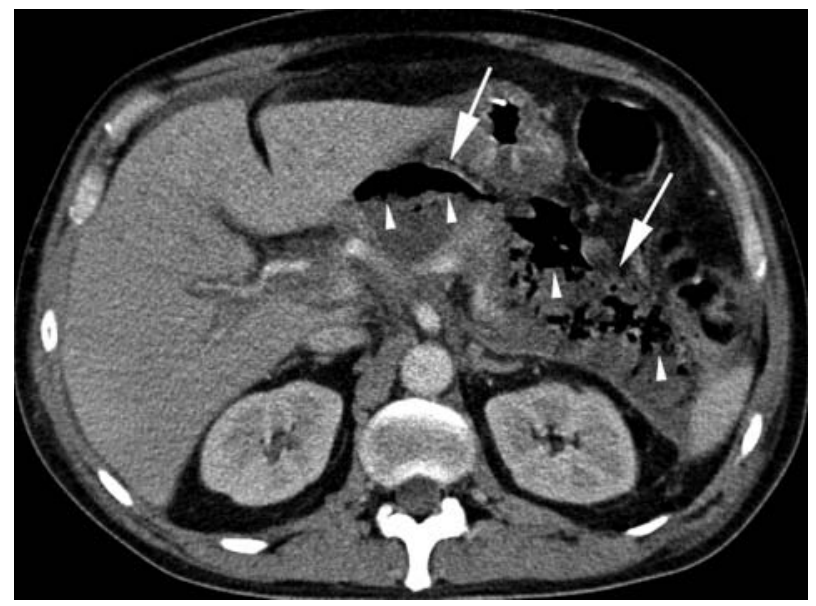

Figure 6 A 47-year-old man with acute necrotising pancreatitis complicated by infected pancreatic necrosis. There is a heterogeneous, acute necrotic collection (ANC) in the pancreatic and peripancreatic area (white arrows pointing at the borders of the ANC) with presence of gas bubbles (white arrowheads), usually a pathognomonic sign of infection of the necrosis (infected necrosis).

\section{Phases of acute pancreatitis}

There are two overlapping phases in this dynamic disease process with two peaks of mortality: early and late. ${ }^{34-37}$ The early phase, which usually lasts for the first week, is followed by a second later phase which can run a protracted course from weeks to months. It is helpful to consider these two phases separately.

\section{Early phase}

During the early phase, systemic disturbances result from the host response to local pancreatic injury. This early phase is usually over by the end of the first week but may extend into the second week. Cytokine cascades are activated by the pancreatic inflammation which manifest clinically as the systemic inflammatory response syndrome (SIRS) ${ }^{38-40}$ (box 2). When SIRS is persistent, ${ }^{41} 42$ there is an increased risk of developing organ failure (table 1). The determinant of the severity of acute pancreatitis during the early phase is primarily the presence and duration of organ failure. This is described as 'transient organ failure' if the organ failure resolves within $48 \mathrm{~h}$ or as 'persistent organ failure' if organ failure persists for
$>48$ h. $^{39} 4143$ If organ failure affects more than one organ system, it is termed multiple organ failure (MOF).

Although local complications may be identified during the early phase, they are not the predominant determinants of severity, ${ }^{32}$ and it may be unreliable to determine the extent of necrosis during the first few days of disease. In addition, the extent of morphologic changes is not directly proportional to the severity of organ failure. ${ }^{24}$ Therefore, the definition of severe or moderately severe acute pancreatitis in the early phase depends on the presence and duration of organ failure (see below, Definition of severity of acute pancreatitis).

\section{Late phase}

The late phase is characterised by persistence of systemic signs of inflammation or by the presence of local complications, and so by definition (see below), the late phase occurs only in patients with moderately severe or severe acute pancreatitis. Local complications evolve during the late phase. It is important to distinguish the different morphologic characteristics of the local complications by radiologic imaging, because these local complications may have direct implications for management. Persistent organ failure, however, remains the main determinant of severity, so

Table 1 Modified Marshall scoring system for organ dysfunction

\begin{tabular}{|c|c|c|c|c|c|}
\hline \multirow[b]{2}{*}{ Organ system } & \multicolumn{5}{|l|}{ Score } \\
\hline & 0 & 1 & 2 & 3 & 4 \\
\hline Respiratory $\left(\mathrm{PaO}_{2} / \mathrm{FiO}_{2}\right)$ & $>400$ & $301-400$ & $201-300$ & $101-200$ & $\leq 101$ \\
\hline \multicolumn{6}{|l|}{ Renal $^{*}$} \\
\hline (serum creatinine, $\mu \mathrm{mol} / \mathrm{l}$ ) & $\leq 134$ & $134-169$ & $170-310$ & $311-439$ & $>439$ \\
\hline (serum creatinine, $\mathrm{mg} / \mathrm{dl}$ ) & $<1.4$ & $1.4-1.8$ & $1.9-3.6$ & $3.6-4.9$ & $>4.9$ \\
\hline Cardiovascular (systolic blood pressure, $\mathrm{mm} \mathrm{Hg}$ ) $†$ & $>90$ & $<90$, fluid responsive & $<90$, not fluid responsive & $<90, \mathrm{pH}<7.3$ & $<90, \mathrm{pH}<7.2$ \\
\hline \multicolumn{6}{|c|}{ For non-ventilated patients, the $\mathrm{FiO}_{2}$ can be estimated from below: } \\
\hline Supplemental oxygen (l/min) & $\mathrm{FiO}_{2}(\%)$ & & & & \\
\hline Room air & 21 & & & & \\
\hline 2 & 25 & & & & \\
\hline 4 & 30 & & & & \\
\hline $6-8$ & 40 & & & & \\
\hline 9-10 & 50 & & & & \\
\hline
\end{tabular}

A score of 2 or more in any system defines the presence of organ failure.

${ }^{*}$ A score for patients with pre-existing chronic renal failure depends on the extent of further deterioration of baseline renal function. No formal correction exists for a baseline serum creatinine $\geq 134 \mu \mathrm{mol} / \mathrm{l}$ or $\geq 1.4 \mathrm{mg} / \mathrm{dl}$.

tOff inotropic support. 
Box 1 Revised definitions of morphological features of acute pancreatitis

1. Interstitial oedematous pancreatitis

Acute inflammation of the pancreatic parenchyma and peripancreatic tissues, but without recognisable tissue necrosis CECT criteria

- Pancreatic parenchyma enhancement by intravenous contrast agent

- No findings of peripancreatic necrosis (see below)

- See figures 1 and 2

2. Necrotising pancreatitis

Inflammation associated with pancreatic parenchymal necrosis and/or peripancreatic necrosis

CECT criteria

- Lack of pancreatic parenchymal enhancement by intravenous contrast agent and/or

- Presence of findings of peripancreatic necrosis (see below-ANC and WON)

- See figures 3, 4, 5 and 8

3. APFC (acute peripancreatic fluid collection)

Peripancreatic fluid associated with interstitial oedematous pancreatitis with no associated peripancreatic necrosis. This term applies only to areas of peripancreatic fluid seen within the first 4 weeks after onset of interstitial oedematous pancreatitis and without the features of a pseudocyst.

CECT criteria

- Occurs in the setting of interstitial oedematous pancreatitis

- Homogeneous collection with fluid density

- Confined by normal peripancreatic fascial planes

- No definable wall encapsulating the collection

- Adjacent to pancreas (no intrapancreatic extension)

- See figure 2

4. Pancreatic pseudocyst

An encapsulated collection of fluid with a well defined inflammatory wall usually outside the pancreas with minimal or no necrosis. This entity usually occurs more than 4 weeks after onset of interstitial oedematous pancreatitis to mature.

CECT criteria

- Well circumscribed, usually round or oval

- Homogeneous fluid density

- No non-liquid component

- Well defined wall; that is, completely encapsulated

- Maturation usually requires $>4$ weeks after onset of acute pancreatitis; occurs after interstitial oedematous pancreatitis

- See figure 7

5. ANC (acute necrotic collection)

A collection containing variable amounts of both fluid and necrosis associated with necrotising pancreatitis; the necrosis can involve the pancreatic parenchyma and/or the peripancreatic tissues

CECT criteria

- Occurs only in the setting of acute necrotising pancreatitis

- Heterogeneous and non-liquid density of varying degrees in different locations (some appear homogeneous early in their course)

- No definable wall encapsulating the collection

- Location-intrapancreatic and/or extrapancreatic

- See figures 3-5

\section{Box 1 (continued)}

6. WON (walled-off necrosis)

A mature, encapsulated collection of pancreatic and/or peripancreatic necrosis that has developed a well defined inflammatory wall. WON usually occurs $>4$ weeks after onset of necrotising pancreatitis.

CECT criteria

- Heterogeneous with liquid and non-liquid density with varying degrees of loculations (some may appear homogeneous)

- Well defined wall, that is, completely encapsulated

- Location-intrapancreatic and/or extrapancreatic

- Maturation usually requires 4 weeks after onset of acute necrotising pancreatitis

- See figure 8

characterisation of acute pancreatitis in the late phase requires both clinical and morphologic criteria.

The SIRS of the early phase may be followed by a compensatory, anti-inflammatory response syndrome (CARS) that may contribute to an increased risk of infection; however, these events are complex and poorly understood. ${ }^{44}$

\section{Definition of severity of acute pancreatitis}

There are important reasons to define and stratify the severity of acute pancreatitis. First, on admission, it is important to identify patients with potentially severe acute pancreatitis who require aggressive early treatment. Second, in a secondary care setting, clinicians need to identify such patients for possible transfer to specialist care. Third, for specialists who receive such referrals, there are advantages to stratifying these patients into subgroups based on the presence of persistent organ failure and local or systemic complications.

This classification defines three degrees of severity: mild acute pancreatitis, moderately severe acute pancreatitis, and severe acute pancreatitis. ${ }^{32}{ }^{33}$ Terminology that is important in this classification includes transient organ failure, persistent organ failure, and local or systemic complications (boxes 1 and 3). Transient organ failure is organ failure that is present for $<48 \mathrm{~h}$. Persistent organ failure is defined as organ failure that persists for $>48 \mathrm{~h}$. Local complications include peripancreatic fluid collections and acute necrotic collections ${ }^{13} 143941$ (box 1), while systemic complications can be related to exacerbations of underlying co-morbidities related to the acute pancreatitis.

\section{Mild acute pancreatitis}

Mild acute pancreatitis is characterised by the absence of organ failure and the absence of local or systemic complications. Patients with mild acute pancreatitis will usually be discharged during the early phase. Patients with mild acute pancreatitis usually do not require pancreatic imaging, and mortality is very rare. ${ }^{15}$

\section{Moderately severe acute pancreatitis}

Moderately severe acute pancreatitis is characterised by the presence of transient organ failure or local or systemic complications in the absence of persistent organ failure. An example of a symptomatic local complication is a peripancreatic collection resulting in prolonged abdominal pain, leucocytosis and 
Box 2 Signs of systemic inflammatory response syndrome (SIRS)

SIRS—-defined by presence of two or more criteria:

- Heart rate $>90$ beats $/ \mathrm{min}$

- Core temperature $<36^{\circ} \mathrm{C}$ or $>38^{\circ} \mathrm{C}$

- White blood count $<4000$ or $>12000 / \mathrm{mm}^{3}$

- Respirations $>20 / \mathrm{min}$ or $\mathrm{PCO}_{2}<32 \mathrm{~mm} \mathrm{Hg}{ }^{13}$

fever, or that prevents the ability to maintain nutrition orally. An example of a symptomatic systemic complication is exacerbation of coronary artery disease or chronic lung disease precipitated by the acute pancreatitis. Moderately severe acute pancreatitis may resolve without intervention (as in transient organ failure or acute fluid collection) or it may require prolonged specialist care (as in extensive sterile necrosis without organ failure). Mortality of moderately severe acute pancreatitis is far less than that of severe acute pancreatitis. ${ }^{32}$

\section{Severe acute pancreatitis}

Severe acute pancreatitis is characterised by persistent organ failure. ${ }^{39}{ }^{41}$ Organ failure that develops during the early phase is set in motion by the activation of cytokine cascades resulting in SIRS 383940 (box 2). When SIRS is present and persistent, ${ }^{39} 4142$ there is an increased risk that the pancreatitis will be complicated by persistent organ failure, and the patient should be treated as if they have severe acute pancreatitis.

Persistent organ failure may be single or multiple organ failure. Patients with persistent organ failure usually have one or more local complications. Patients who develop persistent organ failure within the first few days of the disease are at increased risk of death, with a mortality reported to be as great as $36-50 \%{ }^{38} 3941$ The development of infected necrosis among patients with persistent organ failure is associated with an extremely high mortality. ${ }^{22} 28$

\section{Evolution of severity of acute pancreatitis}

At admission, mild pancreatitis is identified by the absence of organ failure. When organ failure is present within the first $24 \mathrm{~h}$ (and organ failure that occurs during the first week of acute pancreatitis is usually present on admission to hospital),

\section{Box 3 Grades of severity}

Mild acute pancreatitis

- No organ failure

- No local or systemic complications

- Moderately severe acute pancreatitis

- Organ failure that resolves within $48 \mathrm{~h}$ (transient organ failure) and/or

- Local or systemic complications without persistent organ failure

- Severe acute pancreatitis

- Persistent organ failure (>48 h)

-Single organ failure

-Multiple organ failure it may be difficult to determine the final grade of severity, because it is not known whether the patient will prove to have transient or persistent organ failure; the patient does not have mild pancreatitis and should be classified and treated initially as potentially having severe acute pancreatitis. If the organ failure resolves within $48 \mathrm{~h}$ (indicating only transient organ failure), the patient should be classified as having moderately severe acute pancreatitis. If the patient develops persistent organ failure, they should be classified as having severe acute pancreatitis. ${ }^{39} 45$ During the early phase, the severity of acute pancreatitis can be reassessed on a daily basis while the pancreatitis is still evolving. Convenient time points to re-evaluate are $24 \mathrm{~h}, 48 \mathrm{~h}$ and 7 days after admission to hospital.

While local complications may be identified during the early phase, it is generally not necessary to document local complications by imaging during the first week. The reasons for this are as follows. First, the presence and extent of pancreatic and peripancreatic necrosis may not be defined clearly on imaging during the first few days of disease. ${ }^{16}$ When necessary, a CECT 5-7 days after admission is more reliable in establishing the presence and extent of pancreatic necrosis. Second, the extent of morphologic changes and necrosis is not directly proportional to the severity of organ failure. ${ }^{24} 46$ Third, even if imaging during the first week identifies the presence of peripancreatic fluid collections or pancreatic necrosis, in general no treatments are required for these conditions at that time.

In the late phase of moderately severe or severe acute pancreatitis, local complications evolve more fully, although some patients with persistent organ failure may recover without local complications. ${ }^{39}$ The presence of infection within areas of necrosis is a marker of increased risk of death. Infected necrosis without persistent organ failure, however, has a lesser mortality rate than infected necrosis with persistent organ failure. A systematic review ${ }^{33}$ found 11 deaths (11\%) in 93 patients with infected necrosis without organ failure and led to the suggestion of a four-tier grading of severity. ${ }^{47}$ Analysis of two large national studies from the Netherlands ${ }^{25} 48$ shows five deaths $(6 \%)$ in 84 patients with infected necrosis without organ failure.

It is important to distinguish the different morphologic characteristics of the local complications, because these local complications may require a variety of interventions to avoid a fatal outcome.

Patients with moderately severe and severe acute pancreatitis can be described more precisely and stratified for the purpose of clinical studies by the nature and number of morphologic and clinical features (box 1 and 3). The descriptors are local complications (absent, sterile or infected) and persistent organ failure (single or multiple). ${ }^{28}{ }^{33}$ Use of these terms will aid clear communication and will focus attention towards the problems that require management in each case.

\section{Definition of pancreatic and peripancreatic collections}

In the present classification, an important distinction is made between collections that are composed of fluid alone versus those that arise from necrosis and contain a solid component (and which may also contain varying amounts of fluid). Below, we define the following terms: acute peripancreatic fluid collection (APFC) occurring in interstitial oedematous pancreatitis; pancreatic pseudocyst as a delayed (usually $>4$ weeks) complication of interstitial oedematous pancreatitis; and necrosis, which may be an acute necrotic collection (ANC, in the early phase and before demarcation) or walled-off necrosis (WON), which is surrounded by a radiologically identifiable capsule (which rarely develops before 4 weeks have elapsed from onset of pancreatitis). 


\section{Acute peripancreatic fluid collection}

Fluid collections usually develop in the early phase of pancreatitis. ${ }^{49}$ On CECT, APFCs do not have a well defined wall, are homogeneous, are confined by normal fascial planes in the retroperitoneum, and may be multiple (figure 2). Most acute fluid collections remain sterile and usually resolve spontaneously without intervention. ${ }^{19}{ }^{49}$ When a localised APFC persists beyond 4 weeks, it is likely to develop into a pancreatic pseudocyst (see below), although this is a rare event in acute pancreatitis. APFCs which resolve or remain asymptomatic do not require treatment and do not by themselves constitute severe acute pancreatitis.

\section{Pancreatic pseudocyst}

The term pancreatic pseudocyst refers specifically to a fluid collection in the peripancreatic tissues (occasionally it may be partly or wholly intra-pancreatic). A pancreatic pseudocyst is surrounded by a well defined wall and contains essentially no solid material (figure 7). Diagnosis can be made usually on these morphologic criteria. If aspiration of cyst content is performed, there is usually a markedly increased amylase activity. A pancreatic pseudocyst is thought to arise from disruption of the main pancreatic duct or its intra-pancreatic branches without any recognisable pancreatic parenchymal necrosis; this theory suggests that consequent leakage of pancreatic juice results in a persistent, localised fluid collection, usually after more than 4 weeks. When there is evident solid necrotic material within a largely fluid-filled cavity, the term pseudocyst should not be used. The development of a pancreatic pseudocyst is extremely rare in acute pancreatitis, and thus the term pancreatic pseudocyst in the setting of acute pancreatitis may fall into disuse. In this classification, pseudocyst does not result from an ANC (defined below). Although CECT is the imaging modality used most commonly to describe pseudocysts, MRI or ultrasonography may be required to confirm the absence of solid content in the collection.

A pseudocyst may also arise in the setting of acute necrotising pancreatitis as a result of a 'disconnected duct syndrome', whereby pancreatic parenchymal necrosis of the neck or body of the gland isolates a still viable distal pancreatic remnant. ${ }^{50}$ A pseudocyst may be evident many weeks after operative necrosectomy due to localised leakage of the disconnected duct into the necrosectomy cavity. Necrosis is absent because it has been removed by the earlier necrosectomy.

\section{Acute necrotic collection}

During the first 4 weeks, a collection containing variable amounts of fluid and necrotic tissue is termed an ANC (figures $3,4,5)$ to distinguish it from an APFC. The necrosis can involve the pancreatic parenchyma and/or the peripancreatic tissues. On CECT, acute pancreatic or peripancreatic necrotic collections contain varying amounts of solid necrotic material and fluid, may be multiple, and may appear loculated. An ANC is not an APFC, because an ANC arises from necrotising pancreatitis (necrosis of the pancreatic parenchyma and/or peripancreatic tissues) and contains necrotic tissue. An ANC may be associated with disruption of the main pancreatic duct within the zone of parenchymal necrosis and can become infected.

Sequential imaging may be useful to characterise acute collections. Within the first week of the disease, it may be difficult to differentiate an APFC from an ANC. At this stage, both types of collections may appear as areas with fluid density (figure 3). After the first week, the distinction between these
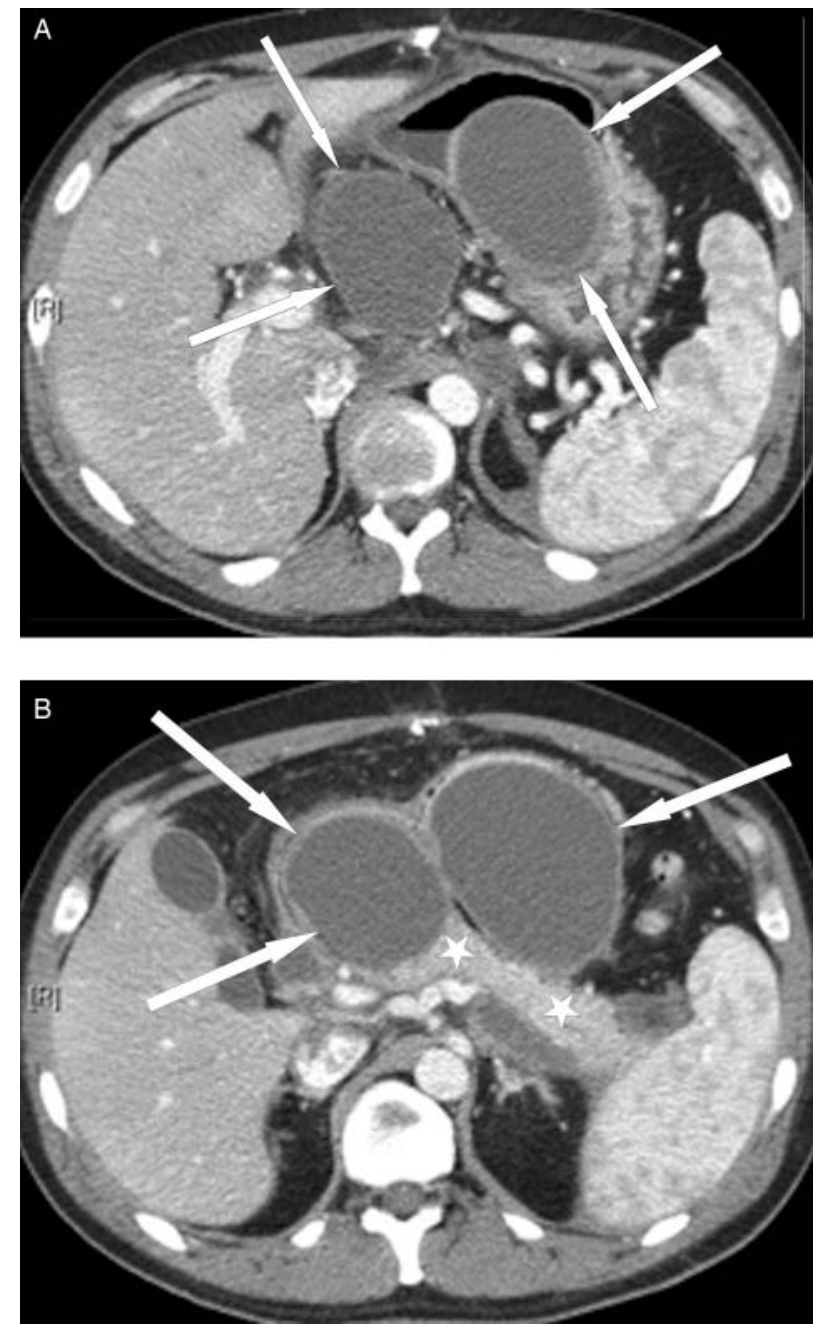

Figure 7 A 40-year-old man with two pseudocysts in the lesser sac 6 weeks after an episode of acute interstitial pancreatitis on CT (A, B). Note the round to oval, low-attenuated, homogeneous fluid collections with a well defined enhancing rim (white arrows pointing at the borders of the pseudocysts), but absence of areas of greater attenuation indicative of non-liquid components. White stars denote normal enhancing pancreas.

two important types of collections becomes clear, such that at this stage of necrosis, a peripancreatic collection associated with pancreatic parenchymal necrosis can be properly termed an ANC and not an APFC. MRI, transcutaneous ultrasonography or endoscopic ultrasonography may be helpful to confirm the presence of solid content in the collection.

\section{Walled-off necrosis}

WON consists of necrotic tissue contained within an enhancing wall of reactive tissue. It is a mature, encapsulated collection of pancreatic and/or peripancreatic necrosis and has a well defined inflammatory wall (figure 8); usually this maturation occurs $\geq 4$ weeks after onset of necrotising pancreatitis. Previous suggested nomenclature had designated this entity as organised pancreatic necrosis, ${ }^{51}$ necroma, ${ }_{54}^{52}$ pancreatic sequestration, ${ }^{53}$ pseudocyst associated with necrosis, ${ }^{54}$ and subacute pancreatic necrosis. ${ }^{55}$

WON derives from necrotic pancreatic parenchyma and/or necrotic peripancreatic tissues and may be infected, may be multiple, and may be present at sites distant from the pancreas. CECT may not readily distinguish solid from liquid content, 
Figure 8 (A-C) Three different patients with walled-off necrosis (WON) after an acute attack of necrotising pancreatitis. In all three patients, a heterogeneous, fully encapsulated collection is noted in the pancreatic and peripancreatic area. (A) Non-liquid components of high attenuation (black arrowheads) in the collection are noted. The collection has a thin, well defined, and enhancing wall (thick white arrows). (B, C) $A$ largely liquefied collection in the bed of the pancreas is observed with non-liquid components representing areas of trapped fat (black arrowheads). (D) represents the corresponding T2-weighted MRI to (C), showing the true heterogeneity of the collection. Black arrowheads denote areas of necrotic debris surrounded by fluid (white on T2-weighted image).
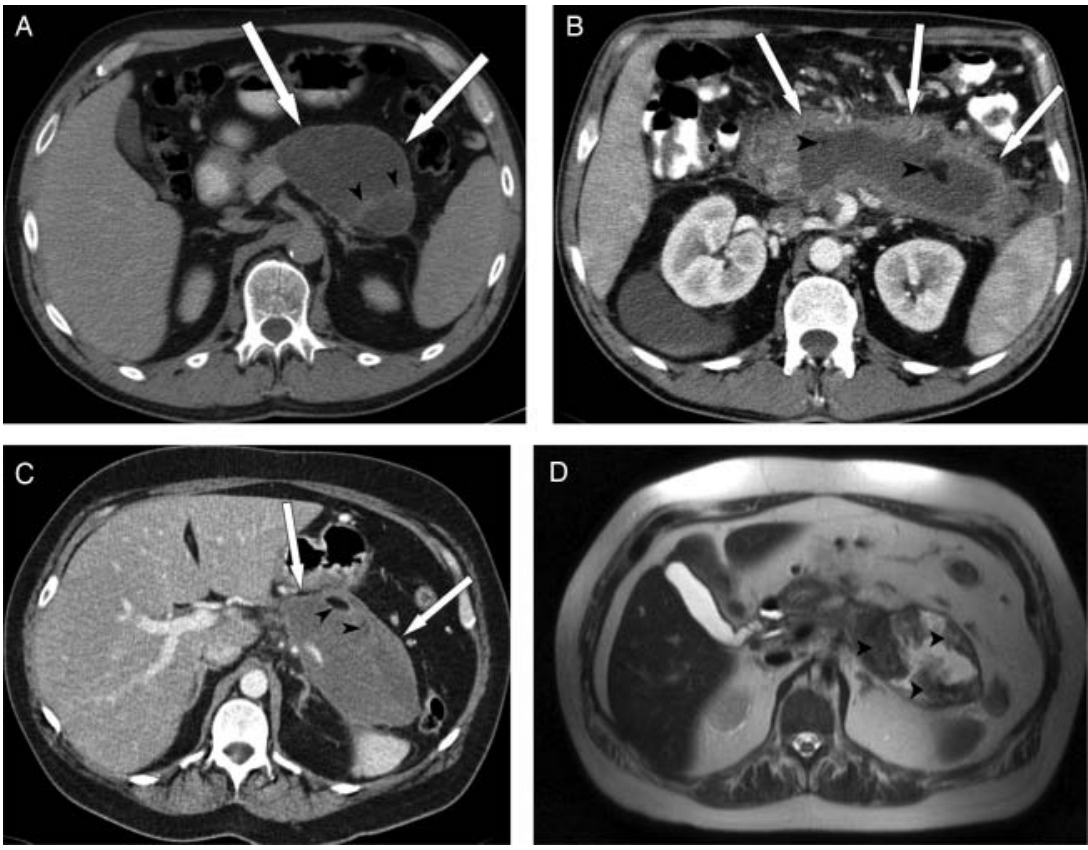

and, for this reason, pancreatic and peripancreatic necrosis may be misdiagnosed as a pancreatic pseudocyst. For this purpose, MRI, transabdominal ultrasonography or endoscopic ultrasonography may be required for this distinction. Demonstration of the presence or absence of pancreatic ductal communication is not necessary in this classification, although determination of such ductal communication is of potential importance, because it may affect management.

\section{Infected necrosis}

The diagnosis of infection (infected necrosis) of an ANC or of WON can be suspected by the patient's clinical course or by the presence of gas within the collection seen on CECT (figure 6). This extraluminal gas is present in areas of necrosis and may or may not form a gas/fluid level depending on the amount of liquid content present at that stage of the disease. In cases of doubt, fine needle aspiration for culture may be performed, but some series have shown that the large majority of patients can be managed without FNA, especially if percutaneous drainage is part of the management algorithm. ${ }^{25}$

\section{CONCLUSION}

This classification revises and updates the definitions from the Atlanta Classification of acute pancreatitis. An important feature is the recognition that acute pancreatitis is an evolving, dynamic condition and that the severity may change during the course of the disease. Early in the disease, SIRS or organ failure indicate potentially severe disease. If the patient improves rapidly during the early phase without organ failure and without local or systemic complications, the disease is defined as mild acute pancreatitis. If the patient develops local or systemic complications and has no persistent organ failure, the disease is defined as moderately severe acute pancreatitis. If the patient develops persistent organ failure, the disease is defined as severe acute pancreatitis and is associated with very high morbidity and mortality rates.

The accurate description of local complications, including the presence of fluid or necrosis in or around the pancreas, the time course of progression, and the presence or absence of infection, will improve the stratification of patients both for clinical care in specialised centres and for reporting of clinical research.

Acknowledgements The Working Group wishes to thank: Deborah Frank for her expert assistance in organising and coordinating electronically all the communications between national and international societies, collating the suggestions made by multiple readers of this new classification on its three internet-based mailings, and finally in the preparation of this manuscript; Drs John A. Windsor, Karen D. Horvath, Koenraad J. Mortele, Timothy B. Gardner, Georgios Papachristou, Pramod Garg, Marc Besselink, Hjalmar van Santvoort, Mario Pelaez-Luna, Dhiraj Yadav, Gerasimos Stefanidis, Stergios Delakidis, Desiree E. Morgan and Ruedi F. L. Thoeni, and the secretaries and other officers of the 11 societies (International Association of Pancreatology (IAP), American Pancreatic Association (APA), European Pancreatic Club (EPC) and from the EPC to its affiliated societies, Pancreatic Disorders Section of the American Gastroenterological Association, Society for Surgery of the Alimentary Tract (SSAT), Pancreas Club, American Hepato-Pancreato-Biliary Association (AHPBA), Japanese Association of

Hepato-Biliary-Pancreatic Surgery, Pancreas Network of New Zealand, Australasian Pancreatic Club, and the Japanese Pancreas Society) who facilitated the dissemination of draft documents; and the following people who responded to one or more of the three working drafts circulated via the web-based process: Acosta, Juan M.; Amann, Stephen T.; Andren-Sandberg, Ake; Aranha, Gerard V.; Asciutti, Stefania; Banks, Peter A.; Barauskas, Giedrius; Baron, Todd. H. Sr; Bassi, Claudio; Behrman, Steven; Behrns, Kevin E.; Belliappa, Vikram; Berzin, Tyler M.; Besselink, Marc G.H.; Bhasin, Deepak Kumar; Biankin, Andrew; Bishop, Michele D.; Bollen, Thomas L.; Bonini, Claudio J.; Bradley. Edward L. III; Buechler, Markus; Carter, Ross; Cavestro, Giulia Martina; Chari, Suresh T.; Chavez-Rodriguez, Juan J.; da Cunha, Jose Eduardo; D’Agostino, Horatio; De Campos, Tercio; Delakidis, Stergios; de-Madaria, Enrique; Deprez, Pierre H.; Dervenis, Christos; DiSario, James A.; Doria, Cataldo; Falconi, Massimo; Fernandez-del Castillo, Carlos; Freeny, Patrick C.; Frey, Charles F.; Friess, Helmut; Frossard, Jean Louis; Fuchshuber, Pascal; Gallagher, Scott F.; Gardner, Timothy B.; Garg, Pramod Kumar; Ghattas, Georges; Glasgow, Robert; Gonzalez, Jose A.; Gooszen, Hein G.; Gress, Thomas M.; Gumbs, Andrew A.; Hallibruton, Cory; Helton, Scott; Hill, Michael C.; Horvath, Karen D.; Hoyos, Sergio; Imrie, Clem W.; Isenmann, Ranier; Izbicki, Jakob R.; Johnson, Colin D.; Karagiannis, John A.; Klar, Ernst; Kolokythas, Orpheus; Lau, Joseph; Litvin, Andrey A.; Longnecker, Daniel S.; Lowenfels, Albert B.; Mackey, Richard; Mah'Moud, Mitchell; Malangoni, Mark; McFadden, David W.; Mishra, Girish; Moody, Frank G.; Morgan, Desiree E.; Morinville, Veronique; Mortele, Koenraad J.; Neoptolemos, John P.; Nordback, Isto; Pap, Akos; Papachristou, Georgios l.; Parks, Rowan; Pedrazolli, Sergio; Pelaez-Luna, Mario; Pezzilli, Raffaele; Pitt, Henry A.; Prosanto, C.; Ramesh, H.; Ramirez, Francisco C.; Raper, Steven E.; Rasheed, Ashraf; Reed, Donald N. Jr; Romangnuolo, Joseph; Rossaak, Jeremy; Sanabria, Juan; Sarr, Michael G.; Schaefer, Claus; Schmidt, Johannes; Schmidt, Palle Nordblad; Serrablo, Alejandro; Senkowski, Christopher K.; Sharma, Manik; Sigman, Ken M.; Singh, Pankaj; Stefanidis, Gerasimos; Steinberg, William; Steiner, Joerg; Strasberg, Steven; Strum, Williamson; Takada, Tadahiro; Tanaka, Masao; Thoeni, Ruedi F. L.; Tsiotos, Gregory G.; Van Santvoort, Hjalmar; Vaccaro, Maria; Vege, Santhi Swaroop; Villavicencio, Roberto L.; 
Vrochides, Dionisios; Wagner, Markus; Warshaw, Andrew L.; Wilcox, Charles M.; Windsor, John A.; Wysocki, Peter; Yadav, Dhiraj; Zenilman, Michael E.; Zyromski, Nicholas J.

Contributions Originators and coordinators (MGS, PAB, SVV). Writing committee (present authors - draft prepared by MGS, PAB, CDJ, and reviewed/commented on by all). Review of drafts - invited expert review; those named below for final review. Individual comments on drafts (long list of responders)

Competing interests None.

Provenance and peer review Not commissioned; externally peer reviewed.

\section{REFERENCES}

1. Bradley EL III. A clinically based classification system for acute pancreatitis. Summary of the International Symposium on Acute Pancreatitis, Atlanta, GA, September 11 through 13, 1992. Arch Surg 1993;128:586-90.

2. Bollen TL, van Santvoort HC, Besselink MG, et al. The Atlanta classification of acute pancreatitis revisited. Br J Surg 2008;95:6-21.

3. Acute Pancreatitis Classification Working Group. Revision of the Atlanta classification of acute pancreatitis. 2008. http://www.pancreasclub.com/resources/ AtlantaClassification.pdf

4. Harrison S, Kakade M, Varadarajula S, et al. Characteristics and outcomes of patients undergoing debridement of pancreatic necrosis. J Gastrointest Surg 2010;14:245-51

5. Brun A, Agarwal N, Pitchumoni CS. Fluid collections in and around the pancreas in acute pancreatitis. J Clin Gastroenterol 2011;45:614-25.

6. Bharwani N, Patel S, Prabhudesai $S$, et al. Acute pancreatitis: the role of imaging in diagnosis and management. Clin Radiol 2011;66:164-75.

7. Sheu Y, Furlan A, Almusa 0, et al. The revised Atlanta classification for acute pancreatitis: a CT imaging guide for radiologists. Emerg Radiol 2012;19:37-43.

8. Thoeni RF. The revised Atlanta classification of acute pancreatitis: its importance for the radiologist and its effect on treatment. Radiology 2012;262:751-64.

9. Banks PA, Freeman ML. Practice guidelines in acute pancreatitis. Am J Gastroenterol 2006;101:2379-400.

10. UK Working Party on Acute Pancreatitis. UK guidelines for the management of acute pancreatitis. Gut 2005;54:iii1-9.

11. Uhl W, Warshaw A, Imrie C, et al. IAP Guidelines for the surgical management of acute pancreatitis. Pancreatology 2002;2:565-73.

12. Arvanitakis $\mathbf{M}$, Delhaye $\mathrm{M}$, De MV, et al. Computed tomography and magnetic resonance imaging in the assessment of acute pancreatitis. Gastroenterology 2004;126:715-23.

13. Bollen TL, van Santvoort HC, Besselink MG, et al. Update on acute pancreatitis: ultrasound, computed tomography, and magnetic resonance imaging features. Semin Ultrasound CT MRI 2007;28:371-83.

14. Morgan DE. Imaging of acute pancreatitis and its complications. Clin Gastroenterol Hepatol 2008;6:1077-85.

15. Singh VK, Bollen TL, Wu BU, et al. An assessment of the severity of interstitial pancreatitis. Clin Gastroenterol Hepatol 2011;9:1098-103.

16. Spanier BWM, Nio Y, van der Hulst RWN, et al. Practice and yield of early CT scan in acute pancreatitis: a Dutch observational multicenter study. Pancreatology 2010;10:222-8.

17. Bollen TL, Singh VK, Maurer $\mathrm{R}$, et al. A comparative evaluation of radiologic and clinical scoring systems in the early prediction of severity of acute pancreatitis. Am J Gastroenterol 2012;107:612-19.

18. Isenmann R, Buechler M, Uhl W, et al. Pancreatic necrosis: an early finding in severe acute pancreatitis. Pancreas 1993;8:358-61.

19. Balthazar EJ, Robinson DL, Megibow AJ, et al. Acute pancreatitis: value of CT in establishing prognosis. Radiology 1990;174:331-6.

20. Sakorafas GH, Tsiotos GG, Sarr MG. Extrapancreatic necrotizing pancreatitis with viable pancreas: a previously under-appreciated entity. J Am Coll Surg 1999;188:643-8.

21. Besselink MG, van Santvoort HC, Boermeester MA, et al. Timing and impact of infections in acute pancreatitis. Br J Surg 2009;96:267-73.

22. van Santvoort HC, Bakker OJ, Bollen TL, et al. A conservative and minimally invasive approach to necrotizing pancreatitis improves outcome. Gastroenterology 2011;141:1254-63.

23. Beger HG, Bittner R, Block S, et al. Bacterial contamination of pancreatic necrosis. A prospective clinical study. Gastroenterology 1986;91:433-8.

24. Perez A, Whang EE, Brooks DC, et al. Is severity of necrotizing pancreatitis increased in extended necrosis and infected necrosis? Pancreas 2002;25:229-33.

25. van Santvoort HC, Besselink MG, Bakker OJ, et al. A step-up approach or open necrosectomy for necrotizing pancreatitis (PANTER trial). N Engl J Med 2010;362:1491-502.

26. Banks PA, Gerzof SG, Langevin RE, et al. CT-guided aspiration of suspected pancreatic infection: bacteriology and clinical outcome. Int J Pancreatol 1995;18:265-70.
27. van Santvoort HC, Bollen $\mathrm{TL}$, Besselink MG, et al. Describing peripancreatic collections in severe acute pancreatitis using morphologic terms: an international interobserver agreement study. Pancreatology 2008;8:593-9.

28. Petrov MS, Shanbhag S, Chakraborty M, et al. Organ failure and infection of pancreatic necrosis as determinants of mortality in patients with acute pancreatitis. Gastroenterology 2010;139:813-20.

29. Marshall JC, Cook DJ, Christou NV, et al. Multiple organ dysfunction score: a reliable descriptor of a complex clinical outcome. Crit Care Med 1995;23:1638-52.

30. Vincent JL, Moreno R, Takala J, et al. The SOFA (Sepsis-related Organ Failure Assessment) score to describe organ dysfunction/failure. On behalf of the working group on sepsis-related problems of the European Society of Intensive Care Medicine. Intensive Care Med 1996;22:707-10.

31. Halonen KI, Pettila V, Leppaniemi AK, et al. Multiple organ dysfunction associated with severe acute pancreatitis. Crit Care Med 2002;30:1274-9.

32. Vege SS, Gardner TB, Chari ST, et al. Low mortality and high morbidity in severe acute pancreatitis without organ failure: a case for revising the Atlanta classification to include "moderately severe acute pancreatitis". Am J Gastroenterol 2009;104:710-15.

33. Petrov MS, Windsor JA. Classification of the severity of acute pancreatitis: how many categories make sense? Am J Gastroenterol 2010;105:74-6.

34. McKay CJ, Imrie CW. The continuing challenge of early mortality in acute pancreatitis. Br J Surg 2004;91:1243-4.

35. Renner IG, Savage WT, Pantoja JL, et al. Death due to acute pancreatitis: a retrospective analysis of 405 autopsy cases. Dig Dis Sci 1995;30:1005-18.

36. Widdison AL, Karanja ND. Pancreatic infection complicating acute pancreatitis. $\mathrm{Br} J$ Surg 1993;80:148-54

37. Blum T, Maisonneuve $\mathrm{P}$, Lowenfels $\mathrm{AB}$, et al. Fatal outcome in acute pancreatitis: its occurrence and early prediction. Pancreatology 2001;1:237-41.

38. Buter A, Imrie CW, Carter CR, et al.Dynamic nature of early organ dysfunction determines outcome in acute pancreatitis. Br J Surg 2002;89:298-302.

39. Johnson CD, Abu-Hilal M. Persistent organ failure during the first week as a marker of fatal outcome in acute pancreatitis. Gut 2004;53:1340-4.

40. Muckart DJ, Bhagwanjee S. American College of Chest Physicians/Society of Critical Care Medicine Consensus Conference definitions of the systemic inflammatory response syndrome and allied disorders in relation to critically injured patients. Crit Care Med 1997;25:1789-95.

41. Mofidi R, Duff MD, Wigmore SJ, et al. Association between early systemic inflammatory response, severity of multiorgan dysfunction and death in acute pancreatitis. Br J Surg 2006;93:738-44.

42. Singh VK, Wu BU, Bollen $\mathrm{TL}$, et al. Early systemic inflammatory response syndrome is associated with severe acute pancreatitis. Clin Gastroenterol Hepatol 2009;7:1247-51.

43. Lytras D, Manes K, Triantopoulou C, et al. Persistent early organ failure: defining the high risk group of patients with severe acute pancreatitis. Pancreas 2008:36:249-54.

44. Cobb JP, O'Keefe GE. Injury research in the genomic era. Lancet 2004;363:2076-83.

45. Johnson CD, Kingsnorth AN, Imrie CW, et al. Double blind, randomised, placebo controlled study of a platelet activating factor antagonist, lexipafant, in the treatment and prevention of organ failure in predicted severe acute pancreatitis. Gut 2001;48:62-9.

46. Tenner S, Sica G, Hughes M, et al. Relationship of necrosis to organ failure in severe acute pancreatitis. Gastroenterology 1997:113:899-903.

47. Dellinger EP, Forsmark CE, Layer $\mathrm{P}$, et al. Determinant-based classification of acute pancreatitis severity: an international multidisciplinary consultation. Ann Surg. Published Online First: 24 Sept 2012. doi: 10.1097/SLA.0b013e318256f778.

48. Besselink MG, van Santvoort HC, Buskens E, et al. Probiotic prophylaxis in predicted severe acute pancreatitis: a randomized, double-blind, placebo-controlled trial. Lancet 2008;371:651-9.

49. Lenhart DK, Balthazar EJ. MDCT of acute mild (nonnecrotizing pancreatitis): abdominal complications and fate of fluid collections. Am J Roentgenol 2008;190:643-9.

50. Pelaez-Luna M, Vege SS, Petersen BT, et al. Disconnected pancreatic duct syndrome in severe acute pancreatitis: clinical and imaging characteristics and outcomes in a cohort of 31 cases. Gastrointest Endosc 2008;68:91-7.

51. Baron TH, Thaggard WG, Morgan DE, et al. Endoscopic therapy for organized pancreatic necrosis. Gastroenterology 1996;111:755-64.

52. Bradley EL III. Atlanta redux. Pancreas 2003;26:105-6.

53. Yeo CJ, Sarr MG. Cystic and pseudocystic diseases of the pancreas. Current Probl Surg 1994;XXXI:165-252.

54. Hariri M, Slivka A, Carr-Locke DL, et al. Pseudocyst drainage predisposes to infection when pancreatic necrosis is unrecognized. Am J Gastroenterol 1994:8:1781-4.

55. Petrakis I, Vrachassotakis N, Kogerakis N, et al. Subacute pancreatic necrosis. Panminerva Med 2000;42:279-86. 